\title{
AN EXAMPLE IN DIMENSION THEORY
}

\author{
R. D. ANDERSON AND J. E. KEISLER ${ }^{1}$
}

An example is constructed which justifies the following theorem:

Theorem 2. There exists a set $K \subset E^{n}$ such that, for each positive integer, $s, \operatorname{dim} K=\operatorname{dim} K^{s}=\operatorname{dim} K^{\omega}=n-1$. Here $\operatorname{dim}$ means (inductive) topological dimension $\left[1\right.$, p. 24], $K^{s}$ is the s-fold product of $K$ with itself and $K^{\omega}$ is the denumerable product of $K$ with itself.

This result is in contrast to the known result that if $A, B$ are nonvoid separable metric spaces with $A$ compact and $\operatorname{dim} B>0$ then $\operatorname{dim}(A \times B) \geqq \operatorname{dim} A$, equality holding only if $\operatorname{dim} A=\infty$.

The construction, for arbitrary $n$, is an exercise in the elementary geometry of $E^{n}$ and transfinite induction. However, for $n=1$, a Cantor set or the rationals on the line will serve as an example; for $n=2$, if the requirement that $K \subset E^{n}$ is deleted (or relaxed to $\left.K \subset E^{n+1}\right)$, the rationals in Hilbert space are an example. For $n>2$, the "standard examples" [1, pp. 29,64] of $n$-dimensional spaces contain cells, so that their arbitrary finite products exhibit increase in dimension.

I. Preliminaries. Let $\omega$ denote the set of positive integers. By continuum we mean a compact, connected, metric space. If $A$ is a set, $\#(A)$ denotes the cardinality of $A$. Let $d$ be a minimal well-ordering of the unit interval, $I$; i.e., for $\alpha \in d, c_{\alpha}=\{\beta \in d \mid \beta<\alpha\}$ has the property $\#\left(c_{\alpha}\right)<\#(I)=c$.

A hyperplane of (linear) dimension $s$ in $E^{r}$ (the solution set of $(r-s)$ linearly independent linear equations) is denoted by $H^{s}$ or by $H$. It is well known that the topological dimension, $\operatorname{dim} H^{s}$, is $s$. Let $\tilde{H}$ denote the set of all linear translates of $H$. For $i=1,2$, let $H_{i}$ be a hyperplane of dimension $t_{i}$ in $E^{r} . H_{1}$ and $H_{2}$ are said to be in general position (with respect to each other) if for $H_{i}^{\prime} \in \widetilde{H}_{i}, H_{1}^{\prime} \cap H_{2}^{\prime} \neq \varnothing$ implies $H_{1}^{\prime} \cap H_{2}^{\prime}=H^{t}$ where $t=\max \left[0, t_{1}+t_{2}-r\right]$. If $H_{1}$ and $H_{2}$ are in general position, we say that $H_{1}$ and $\widetilde{H}_{2}$, and that $\widetilde{H}_{1}$ and $\widetilde{H}_{2}$, are in general position.

It is convenient to consider $\left(E^{n}\right)^{s}=E^{n s}$ as all "s-letter words," each letter being a point of $E^{n}$. Let $\tau_{A}:\left(E^{n}\right)^{s} \rightarrow\left(E^{n}\right)^{t}$ be defined by deleting

Presented to the Society, January 24, 1967 ; received by the editors December 16, 1966.

1 The work of the first named author was supported in part by National Science Foundation grants. 
the $j$ th letter of $w \in\left(E^{n}\right)^{s}$ for all $j \in A$, where $A$ is a proper subset of $\omega_{s}=\{j \in \omega \mid 1 \leqq j \leqq s\}$. Then $t=s-\#(A)$. We abbreviate $\tau_{\{j\}}$ as $\tau_{j}$. Let $\theta$ denote the origin in $E^{n}$. For $\varnothing \neq A \subset \omega_{s}$, define $H(A)$ as the $n$-dimensional hyperplane which is the solution set of the equations $\tau_{j}(p)$ $=\tau_{k}(p)$ for $j, k \in A$ and $\tau_{i}(p)=\theta$ for $p \in \omega_{s} \backslash A$. Let $\gamma=\{\widetilde{H}(A) \mid \varnothing$ $\left.\neq A \subset \omega_{s}\right\}$. [Each $\tilde{H}(A)$ may be thought of as a direction for $n$-dimensional hyperplanes. In this sense, if $A=\omega_{s}, \widetilde{I}(A)$ represents the "diagonal" direction; if $A=\{j\}, \widetilde{H}(A)$ represents the direction parallel to the $j$ th coordinate plane.]

The set $S^{k}$ is a $k$-sphere in $E^{r}$ iff $S^{k}=S \cap H^{k+1}$ where $\#\left(S^{k}\right)>1$ and $S$ is an $(r-1)$ sphere; i.e., $S$ is the set of all points at fixed positive distance from a given point of $E^{r}$. Hence, $S^{k}$ is the set of all points in $H^{k+1}$ at fixed distance from a given point of $H^{k+1}$.

Let $M^{k}$ denote a $k$-dimensional Cantor-manifold [1, p. 93].

II. Lemmas. With reference to the notation of Theorem 2, $K$ will be constructed so that the following Lemma 1 is applicable. Thus $\operatorname{dim} K \geqq n-1$ and therefore $\operatorname{dim} K^{s} \geqq n-1$. Lemma 1 is probably known, at least in the folklore.

Lemma 1. Let $K \subset E^{n}$ such that for each nondegenerate continuum $C \subset E^{n}, K \cap C \neq \varnothing$. Then $\operatorname{dim} K \geqq n-1$.

Proof. Choose $p_{0} \in K$ and any $U^{\text {open }} \subset E^{n} \ni p_{0} \in U_{0}=U$ and diam $U<1$. $\bar{U} \backslash U$ separates $E_{n}$, hence it contains an $(n-1)$-dimensional Cantor-manifold, $M^{n-1} ; K \cap(\bar{U} \backslash U) \supset K \cap M^{n-1} \neq \varnothing$ by hypothesis. Hence, $\operatorname{dim}\left(K \cap M^{n-1}\right) \geqq 0$. If $\operatorname{dim}\left(K \cap M^{n-1}\right) \geqq s$ for all $M^{n-1} \subset E^{n}$ then $\operatorname{dim} K \geqq s+1$. Inductively, let $p_{i} \in M^{n-i} \subset E^{n}$ and let $U_{n-i}$ be an open set in $M^{n-i} \ni \bar{U}_{n-i} D M^{n-i}$. Then $\bar{U}_{n-i} \backslash U_{n-i}$ separates $M^{n-i}$ and therefore contains an $(n-i-1)$-dimensional Cantormanifold $M^{n-i-1}, i<n$. If $\operatorname{dim}\left(K \cap M^{n-i-1}\right) \geqq s$ for all such $M^{n-i-1}$ $\subset E^{n}$ then $\operatorname{dim}\left(K \cap M^{n-i}\right)>s+1$. But for each $M^{1} \subset E^{n}, K \cap M^{1} \neq \varnothing$. Therefore $\operatorname{dim}\left(K \cap M^{1}\right) \geqq 0 \Rightarrow \operatorname{dim}\left(K \cap M^{2}\right) \geqq 1 \Rightarrow \cdots \operatorname{dim}\left(K \cap M^{n-1}\right)$ $\geqq n-2 \Rightarrow \operatorname{dim} K \geqq n-1$.

Of course, $\operatorname{dim} K=n$ iff $K$ contains a nonnull open subset of $E^{n}$.

We shall use, without explicit proof here, a weakened form of the following lemma, which asserts that hyperplanes may be tilted a small amount so that they are moved into general position with respect to a countable set of hyperplanes and continue to separate spheres about as they did before tilting.

Lemma 2. Given a countable collection of families of hyperplanes, $\left\{\tilde{H}_{i}\right\}$, a $k$-sphere $S$, and a hyperplane $H$, all in $E^{r}$, such that $S \backslash H$ $=U_{1} \cup U_{2}$ where $p \in U_{1}, U_{i}$ is open and closed in $S \backslash H$, and $U_{1} \cap U_{2}=\varnothing$. 
Then there exists a hyperplane $H^{\prime}$ such that (1) $\operatorname{dim} H^{\prime}=k$, (2) for each $i \in \omega, H^{\prime}$ is in general position with respect to $\widetilde{H}_{i}$, and (3) $S \backslash H^{\prime}=V_{1} \cup V_{2}$ where $p \in V_{1} \subset U_{1}, V_{i}$ is open and closed in $S \backslash H^{\prime}$, and $V_{1} \cap V_{2}=\varnothing$.

We now introduce some notations for use in Lemma 3 below. Choose a countable dense set of points in $E^{n s}$ and the $(n s-1)$-dimensional spheres $S^{n s-1}$ with rational radii about them. For each $S^{n s-1}$, choose a countable set of $(n s-1)$-dimensional hyperplanes $H^{n-1}$ so that their complementary domains form a basis for the topology of $S^{n s-1}$ and so that, with $\gamma$ defined as in $\S \mathrm{I}$, each $H^{n s-1}$ is in general position with respect to the $\widetilde{H}^{n} \in \gamma$. Lemma 2 assures us that this is possible. On each of the countably many $S^{n s-1}$ 's, we choose countably many $S^{n s-2}$ 's by $S^{n s-2}=S^{n s-1} \cap H^{n s-1}$, for the $H^{n s-1}$ 's chosen above.

Inductively, for each chosen $S^{n s-k}=S^{n s-k+1} \cap H^{n s-k+1}$, choose a countable set of hyperplanes, $H^{n s-k}$, whose complementary domains in $S^{n s-k}$ form a basis for the topology of $S^{n s-k}$ and such that $H^{n s-k}$ is in general position with respect to $\tilde{H}^{n} \in \gamma$. In this way, countably many spheres, $\left\{S_{i}^{t}\right\}$ are chosen, $n s-n \leqq t<n s$. Denote $S_{i}^{n s-n}$ by $S_{\boldsymbol{i}}$.

Lemma 3. Let $T \subset E^{n s}$ such that, for each $i, T \cap S_{i}=\varnothing$, the spheres being chosen as above. Then $\operatorname{dim} T \leqq n-1$.

Proof. The complementary domains of the $S_{i}^{n s-k}$ in the $S_{i}^{n s-k+1}$ form a topological basis for $S^{n-k+1}$, by construction. Therefore, $T \cap S_{i}=\varnothing \Rightarrow \operatorname{dim}\left(T \cap S_{i}\right)=-1 \Rightarrow \operatorname{dim}\left(T \cap S^{n s-n+1}\right) \leqq 0 \Rightarrow \operatorname{dim}\left(T \cap S^{n s-n+2}\right)$ $\leqq 1 \Rightarrow \cdots \Rightarrow \operatorname{dim}\left(T \cap S^{n s-1}\right) \leqq n-2 \Rightarrow \operatorname{dim} T \leqq n-1$.

Lemma 4. Let $K \subset E^{n} \ni \operatorname{dim} K^{s}<t \forall s \in \omega$. Then $\operatorname{dim} K^{\omega}<t$.

Proof. By $[2$, p. 126], it suffices to show that there is a sequence, $\mathcal{U}_{i}$, of open covers of $K^{\omega} \ni$ :

(i) $\mathfrak{u}_{i+1} \prec \mathfrak{u}_{i}$,

(ii) order $\mathfrak{u}_{i} \leqq t$,

(iii) mesh $u_{i}<1 / 2^{i-2}$.

We consider $K \subset(0,1)^{n} \approx E^{n}$ and $K^{\omega} \subset\left[\left(0_{1}, 1\right)^{n}\right]^{\omega} \subset I^{\omega}$, the Hilbert cube. If $p, q \in I^{\omega}, p=\left\{p_{i}\right\}, q=\left\{q_{i}\right\}$ then $d(p, q)=\sum\left(1 / 2^{i}\right)\left|p_{i}-q_{i}\right|$.

Construct an open cover, $V_{1}$, of $K \subset(0,1)^{n} \ni$ order $V_{1} \leqq t$ and mesh $V_{1}<\frac{1}{2}$. This is possible, since $\operatorname{dim} K<t$. Inductively, assume we have an open cover $V_{i}$ of $K^{i} \subset E^{n i} \ni$ order $V_{i} \leqq t$ and mesh $V_{i}<1 / 2^{i}$. Let $V_{i}^{\prime}=\left\{v \times(0,1)^{n} \mid v \in V_{i}\right\} . V_{i}^{\prime}$ is an open cover of $K^{i+1} \subset E^{n(i+1)}$. Since $\operatorname{dim} K^{i+1}<t, \quad \exists V_{i+1}$, an open cover of $K^{i+1}, \exists V_{i+1} \prec V_{i}^{\prime}$, order $V_{i+1} \leqq t$ and mesh $V_{i+1}<1 / 2^{i+1}$. Let $\mathcal{u}_{i}=\left\{v \times \Pi_{j=n i+1}^{\infty}(0,1)^{j} \mid v \in V_{i}\right\}$. Since $V_{i+1} \prec V_{i}^{\prime}$ we have $u_{i+1} \prec \mathcal{u}_{i}$. Order $\mathcal{u}_{i+1}=$ order $V_{i+1}$ and mesh $u_{i} \leqq$ mesh $V_{i}+2 \sum_{j=n i+1}^{\infty} 1 / 2^{j}<1 / 2^{i}+2 / 2^{n i}<1 / 2^{i-2}$.

Therefore, $\operatorname{dim} K^{\omega}<t$. 


\section{Theorems.}

Theorem 1. Let $n, s \in \omega$. There exists $K \subset E^{n}$ such that $\operatorname{dim} K$ $=\operatorname{dim} K^{s}=n-1$.

Proof. We construct $K$ by transfinite induction. Let $\left\{S_{i}^{r}\right\}_{i \in \omega}$, $n s-n \leqq r<n s$, be constructed as for Lemma 3, for the collection $\gamma$, with $S_{i} \subset H_{i}$ where $\operatorname{dim} H_{i}=n s-n+1$. Let $R=\bigcup S_{i}$. Let $\mathcal{C}=\left\{C_{\alpha}\right\}$, $\alpha \in d$, be a minimal well-ordering of the nondegenerate continua contained in $E^{n}$. Consider $H \in \widetilde{H} \in \gamma, C \in \mathcal{C}$, and $S_{i} \cdot H_{i} \cap H$ is a line, by Lemma 2. Therefore $S_{i} \cap H=S_{i} \cap H \cap H_{i}$ is at most 2 points.

Let $w$ be a $k$-letter word in $E^{n k}, 0 \leqq k<s, x \in E^{n}$ and $w(x)$ the (finite) set of all $s$-letter words in $E^{n s} \ni$ for $w^{\prime} \in w(x) \exists A \subset \omega_{s}$ for which $\tau_{A}\left(w^{\prime}\right)=w$ and $\tau_{j}\left(w^{\prime}\right)=x, j \in \omega_{s} \backslash A$. Let $A(w, \alpha)=\left\{x \mid x \in C_{\alpha}\right.$ and $\left.\exists w^{\prime} \in w(x) \cap R\right\} . A(w, \alpha)$ is a countable set, since $w$ determines a finite set of $H_{j} \in \tilde{H} \in \gamma, \#\left(H \cap S_{i}\right) \leqq 2$, and $A(w, \alpha) \subset \bigcup_{j, i}\left(H_{j} \cap S_{i}\right)$.

Let $L \subset E^{n}$ and $A(L, \alpha)=\left\{x \mid x \in A(w, \alpha), w \in L^{k}, 0 \leqq k<s\right\}$. If $\#(L)<c$ then $\#[A(L, \alpha)]<c$.

Consider $C_{1}$, the first element of $\mathcal{e}$. Let $H$ be the diagonal $n$-hyperplane in $E^{n s}$. Since $H \cap R$ is countable, there exists $x_{0} \in C_{1} \ni\left(x_{0}\right)^{s} \in R$. Let $K_{1}=\left\{x_{0}\right\}$.

Suppose that, for $\beta<\alpha$, the sets $K_{\beta}$ have been defined so that $\beta_{1}<\beta_{2} \Rightarrow K_{\beta_{1}} \subset K_{\beta_{2}}, \#\left(K_{\beta}\right)<c, C_{\beta} \cap K_{\beta} \neq \varnothing$, and $K_{\beta}^{s} \cap R=\varnothing$. Let $K_{\alpha}^{\prime}$ $=\bigcup_{\beta<\alpha} K_{\beta}$. Then $\#\left(K_{\alpha}^{\prime}\right)<c$ and $\left(K_{\alpha}^{\prime}\right)^{s} \cap R=\varnothing$. If $K_{\alpha}^{\prime} \cap C_{\alpha} \neq \varnothing$, let $K_{\alpha}=K_{\alpha}^{\prime}$. Otherwise, choose (appropriately) a point $p_{\alpha} \in C_{\alpha}$ and let $K_{\alpha}=K_{\alpha}^{\prime} \cup\left\{p_{\alpha}\right\}$. The inductive properties, except $K_{\alpha}^{s} \cap R=\varnothing$, are clearly realized. Since $\#\left(K_{\alpha}^{\prime}\right)<c$, \#A $\left(K_{\alpha}^{\prime}, \alpha\right)<c$. But $\#\left(C_{\alpha}\right)=c$. Therefore, $C_{\alpha} \backslash A\left(K_{\alpha}^{\prime}, \alpha\right) \neq \varnothing$. Hence, choose $p_{\alpha} \in C_{\alpha} \backslash A\left(K_{\alpha}^{\prime}, \alpha\right)$ and $K_{\alpha}$ $=K_{\alpha}^{\prime} \cup\left\{p_{\alpha}\right\}$ satisfies $K_{\alpha}^{s} \cap R=\varnothing$.

Thus, we construct $K_{\alpha} \forall \alpha$. Let $K=\mathrm{U} K_{\alpha}$. Then $K^{s} \cap R=\varnothing$. By Lemma $3, \operatorname{dim} K^{s} \leqq n-1$. Therefore $\operatorname{dim} K \leqq n-1$. But Lemma 1 applies to $K$, by construction. Hence $\operatorname{dim} K \geqq n-1$. This implies that $\operatorname{dim} K^{s} \geqq n-1$. Thus, $\operatorname{dim} K^{s}=n-1$.

A slight variant of the procedure above allows us to prove the following.

THEOREM 2. There exists a set $K \subset E^{n}$ such that for each positive integer, $s, \operatorname{dim} K=\operatorname{dim} K^{s}=\operatorname{dim} K^{\omega}=n-1$.

Proof. For each $s$, we construct the spheres $S_{i}^{n s-n}$ in $E^{n s}$. In the inductive definition of $K$, as above, we assume in addition that $K_{\beta}^{s} \cap S_{i}^{n s-n}=\varnothing$ for each $s$ and $i$. Let $K_{\alpha}^{\prime}=\bigcup_{\beta<\alpha} K_{\beta}$. For $L \subset E^{n}$ and $s \in \omega$, let $A(L, \alpha, s)$ be the set constructed in $E^{n s}$, and denoted by $A(L, \alpha)$, in Theorem 1 . We have $\#\left(A\left(K_{\alpha}^{\prime}, \alpha, s\right)\right)<c$. Hence 
$\#\left(\bigcup_{s \in \omega} A\left(K_{\alpha}^{\prime}, \alpha, s\right)\right)<c$ and $\exists p_{\alpha} \in C_{\alpha} \backslash \cup A\left(K_{\alpha}^{\prime}, \alpha, s\right)$. Let $K_{\alpha}=K_{\alpha}^{\prime}$ $\cup\left\{p_{\alpha}\right\}$. Then $K_{\alpha}$ fulfills the inductive assumptions and $K=\cup K_{\alpha}$ satisfies $\operatorname{dim} K^{s}=n-1$, for each $s \in \omega$. Hence, by Lemma 4, $\operatorname{dim} K^{\omega}<n$. But $K^{\omega} \supset K^{\prime}$ where $K^{\prime} \stackrel{T}{\approx} K$ and $\operatorname{dim} K \geqq n-1$. Thus $\operatorname{dim} K^{\omega}=n-1$.

iv a

\section{REFERENCES}

1. W. Hurewicz and H. Wallman, Dimension theory, Princeton Univ. Press, Princeton, N. J., 1941.

2. J. Nagata, Modern dimension theory, Interscience, New York, 1965.

Louisiana State University

\section{IMMERSIONS INTO MANIFOLDS OF CONSTANT NEGATIVE CURVATURE}

\section{EDSEL F. STIEL ${ }^{1}$}

1. Introduction. Let $M$ and $\bar{M}$ denote $C^{\infty}$ Riemannian manifolds, $K$ and $\bar{K}$ their respective sectional curvature functions, and $\psi: M \rightarrow \bar{M}$ an isometric immersion. A consequence of Theorem 2 of [5] is that if at any point $m \in M, K(\pi)<\bar{K}(d \psi(\pi))$, where $\pi$ is some plane in $M_{m}$, (the tangent space to $M$ at $m$ ) then there are no $\psi$ that immerse $M^{d}$ in $\bar{M}^{d+k}$ unless $k$ is greater than or equal to $d-1$. By restricting $M$ to be compact and $\bar{M}$ to be complete and simply connected, O'Neill has shown in [3] that there are no isometric immersions of $M^{d}$ in $\bar{M}^{d+k}$ when $K \leqq \bar{K} \leqq 0$ on $M$ unless $k$ is greater than or equal to $d$. Amaral (Theorem A of [1]) considered immersions of compact $M^{d}$ in $H^{d+1}(\bar{C})$, $(d+1)$-dimensional hyperbolic space of curvature $\bar{C}$, and by only assuming $K \leqq 0$ proved that there are no isometric immersions of $M^{d}$ in $H^{d+1}(\bar{C})$. Using methods similar to those of [3] we prove a theorem which strengthens O'Neill's result in the case that $\bar{M}$ is of constant negative curvature and includes Amaral's result.

\section{Results.}

TheOREM. Let $M$ be a compact d-dimensional Riemannian manifold and let $\bar{M}$ be a complete simply connected Riemannian manifold of constant curvature $\bar{C} \leqq 0$ and of dimension less than $2 d$. If the sectional

Received by the editors July 13, 1966.

${ }^{1}$ Part of the research in this paper was done while the author was the recipient of an NSF Research Participation award in Mathematics at the University of Oklahoma. 